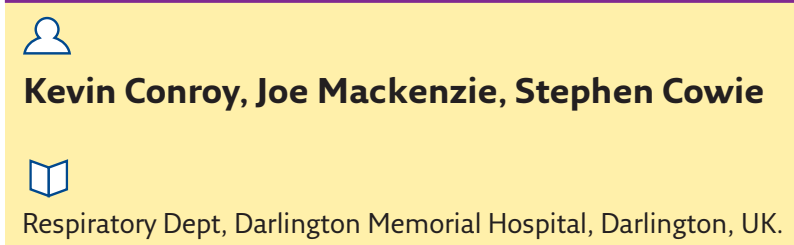

Respiratory Dept, Darlington Memorial Hospital, Darlington, UK.

\title{
Common things are common, but not always the answer
}

\section{Case report}

We reviewed a 76-year-old lady with a persisting left upper lobe opacity on subsequent chest radiographs. She complained of weight loss but denied constitutional upset. Bloods showed raised IgG subclass immunoglobulins. Computed tomography (CT) of the thorax demonstrated consolidation in the left upper lobe and small volume mediastinal adenopathy. Bronchoscopy was negative and CT-guided lung biopsy showed only inflammatory tissue. This was repeated with a similar result. As there was no evidence of malignancy, our patient was commenced on oral steroids and was discharged. Unfortunately, she re-presented 4 years later following an episode of acute dyspnoea. She underwent a further CT of the thorax and CT-guided biopsy in order to rule out malignancy. Storiform fibrosis was noted on histology and a diagnosis of IgG4related lung disease was reached. IgG4-related lung disease had clearly not been considered as a differential diagnosis in this case. We pushed to rule out malignancy but did not explore alternative diagnoses. This highlights the need to consider other conditions or they will be missed!

A 76-year-old lady was referred to the chest clinic with a persisting left upper lobe opacity on subsequent chest radiography (figure 1). She was a current smoker with a history of diverticular

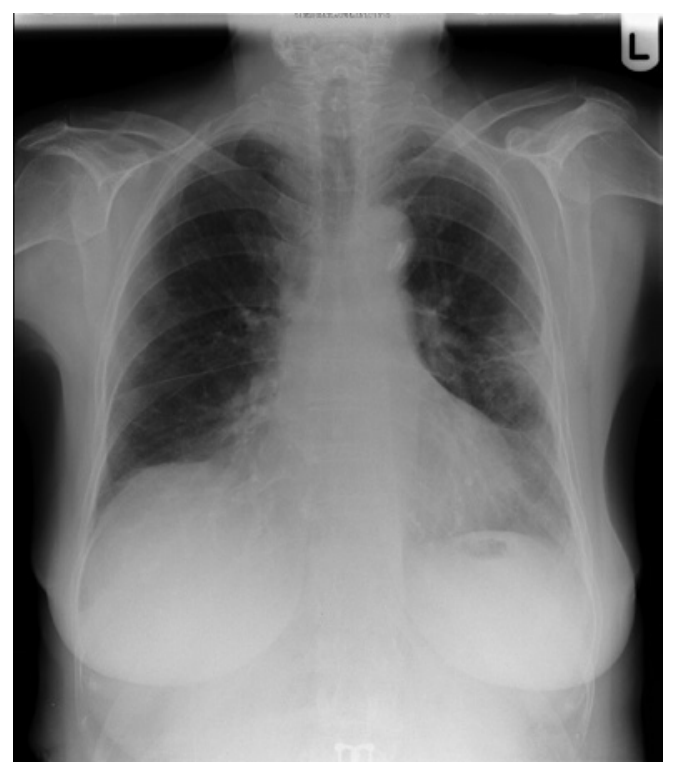

Figure 1 Initial chest radiograph.

disease and childhood tuberculosis exposure. She complained of weight loss but denied haemoptysis, cough, night sweats or constitutional upset. She described exertional dyspnoea consistent with MRCD2. Spirometry in clinic was recorded as a
Cite as: Conroy K, Mackenzie J, Cowie S. Common things are common, but not always the answer. Breathe 2016; 12 : e104-e108. 
forced expiratory volume in $1 \mathrm{~s}$ of $1.34 \mathrm{~L}(66 \%$ predicted) and forced vital capacity of $67 \%$ predicted, consistent with a mild restrictive defect. Her initial blood tests revealed a hypochromic microcytic anaemia, negative inflammatory markers and raised IgG and IgA levels. Serum electrophoresis revealed polyclonal increase in $\gamma$-globulins. A contrast-enhanced staging CT of the thorax demonstrated consolidation in the anterior segment of the left upper lobe and small volume mediastinal adenopathy (figures 2 and 3 ).

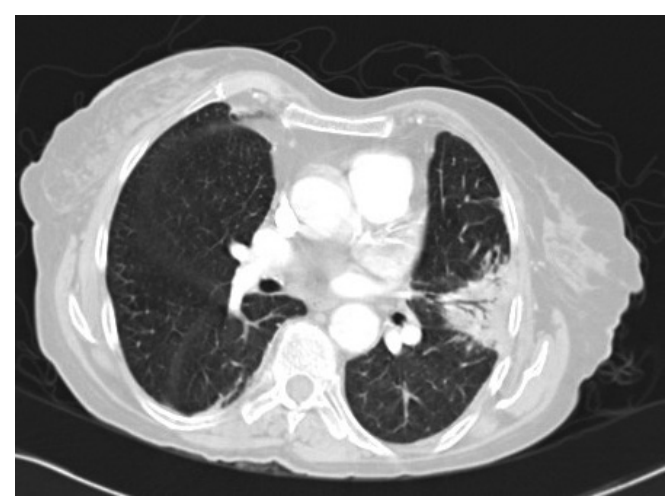

Figure 2 Initial CT lung window.

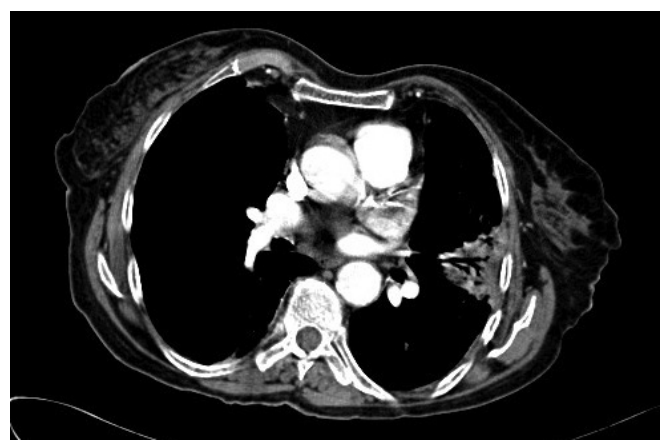

Figure 3 Initial CT of the aortic-pulmonary window.

Task 1

What conditions would be on a list of differential diagnoses at this point?
a. Bacterial pneumonia
b. Tuberculosis
c. Inflammatory consolidation (for example COP, eosinophilic pneumonia, lgG4 disease)
d. Broncioalveolar carcinoma/ adenocarcinoma in situ
e. All of the above 


\section{Answer 1}

e. All of the above

All of the above are reasonable differential diagnoses in a patient with non-segmental consolidation and weight loss. Her smoking history prioritised malignancy as a key differential diagnosis. However neglecting other causes may preclude a timely diagnosis.

\section{Task 2}

Which investigation would you perform next?

a. Bronchoscopy with lavage of left upper lobe

b. CT guided biopsy

c. PET scan

d. Sputum cytology

e. Surgical lung biopsy

\section{Answer 2}

\section{a. Bronchoscopy with lavage of left upper lobe}

Bearing in mind our broad differential and the possibility of an infective cause, bronchoscopy with lavage is the most appropriate first investigation. CT-guided biopsy carries an increased risk compared with bronchoscopy and may not be diagnostic of infection. Positron emission tomography (PET) scanning would not be helpful at this stage, as it is unlikely to help differentiate between the causes. Sputum cytology has a very low yield and is not recommended. Surgical lung biopsy would carry even greater risk than CT-guided biopsy.

Bronchoscopy was unremarkable with negative cultures and no evidence of malignancy on bronchial washings. Following multidisciplinary team discussion adenocarcinoma in situ remained a differential so a CT-guided biopsy was performed. Histology did not show any evidence of malignancy but did show a chronic fibro-inflammatory process.

A plan was made for a repeat CT scan after 3 months as it was felt that the abnormality could be a slowly resolving infection or cryptogenic organising pneumonia. Our patient commenced a trial of oral steroids with some improvement in her general condition. Meanwhile, anaemia of chronic disease was diagnosed.

A repeat CT scan performed at 3 months failed to show any resolution of the consolidation.

\section{Task 3}

There was no change on the interval scan; what are our options now?
a. PET scan
b. Repeat CT-guided biopsy
c. Surgical lung biopsy
d. Second opinion on histology from tertiary centre
e. Serum lgG4 levels 


\section{Answer 3}

$\mathrm{d}$ and e. second opinion on histology from tertiary centre and serum IgG4 levels

By this point, consideration of a nonmalignant diagnosis is appropriate. Serum IgC immunoglobulins were consistently raised and polyclonal $\gamma$-globulins had been recorded. Further analysis of this with immunoglobulin sub-typing would be a suitable course of action. So too would be further analysis of the chronic inflammation found on the CT-guided biopsy. A PET scan is still unlikely to distinguish between malignant and non-malignant diagnosis. A further biopsy is not without risk but is reasonable; particularly if there is suspicion of malignancy. Had options d and e been followed, a diagnosis of IgG4-related lung disease may have been made at this point. Unfortunately this did not happen.

The case was re-discussed at the lung multidisciplinary team and our patient underwent a repeat CT-guided biopsy. Once again this showed a chronic fibro-inflammatory process with no evidence of malignancy. Our patient was reviewed in the clinic 6 months after initial presentation and felt well. Given her stability, she was discharged with no conclusive diagnosis.

\section{Follow up}

Our patient re-presented to the respiratory service 3 years later following a hospital admission with acute dyspnoea, pleuritic chest pain and malaise. A CT pulmonary angiogram ruled out pulmonary embolism but showed persistent consolidation in her left upper lobe and mediastinal adenopathy (figure 4). She was treated for pneumonia and underwent further investigations.

Once again her bloods showed anaemia, raised inflammatory markers (erythrocyte sedimentation

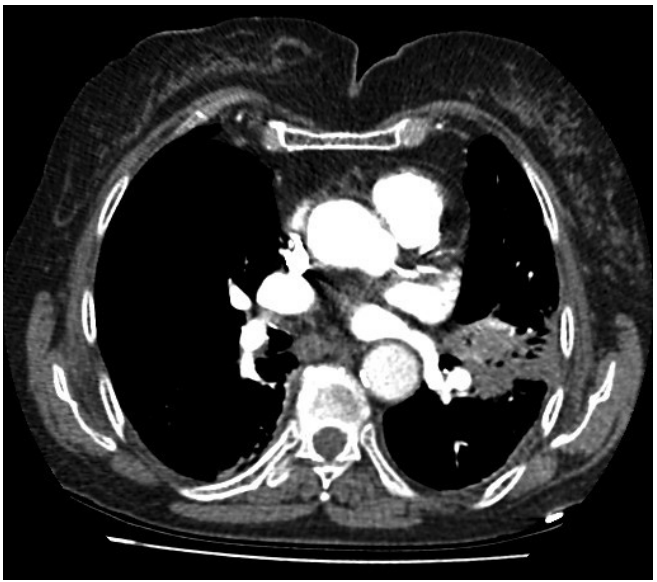

Figure 4 CT pulmonary angiogram. rate of 127), raised IgG and polyclonal increase in $\gamma$-globulins.

A third CT-guided biopsy was undertaken, which again showed fibro-inflammatory cells. The histology was re-examined at a specialist histopathology centre and IgG4-related lung disease was confirmed. Her condition improved with a short course of steroids and, after a 4-year journey, our patient finally received a diagnosis. Her consolidation remains unchanged. A recent chest radiograph is shown in figure 5 .

\section{Discussion}

IgG4-related lung disease can present as adenopathy, consolidation or interstitial disease. It is a rare, idiopathic multi system condition characterised by raised serum IgG4 levels and lgG4-positive lymphoplasmacytic infiltrates and storiform fibrosis on biopsy. The aetiology of IgG4-related disease is poorly understood. Raised serum and tissue $\lg \mathrm{G} 4$ has been noted in other conditions such as sarcoidosis and granulomatous polyangiitis. Symptoms of IgG4-related disease are organ specific, but general symptoms include weight loss, malaise and constitutional upset. It is typically steroid responsive.

\section{Conclusion}

IgG4-related lung disease had clearly not been considered as a differential diagnosis in this case. We pushed to rule out malignancy but did not explore alternative diagnoses. Because of this our patient suffered three invasive and potentially dangerous biopsies over a protracted period. This highlights the need to consider rare conditions or they will be missed!

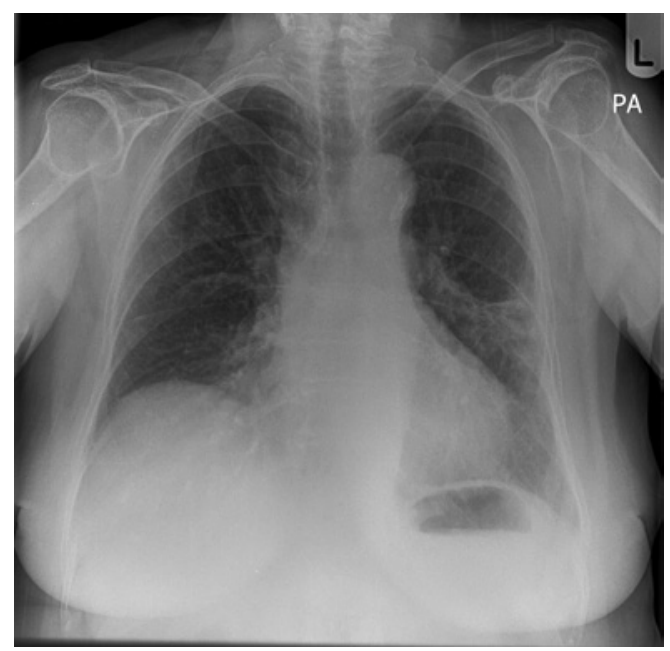

Figure 5 A recent chest radiograph. 


\section{Conflict of interest}

None declared

\section{Further reading}

1. Haroon A, Higa F, Haranaga S, et al. Differential diagnosis of non-segmental consolidations. J Pulmon Respir Med 2013.
2. Stone JH, Zen Y, Deshpande V. IgG4-related disease. N Engl

3. Kamisawa T, Zen Y, Pillai S, et al. IgG4-related disease. Lancet 2015; 385: 1460

Med 2012; 366: 539. 